\title{
Return of symptomatic congenital syphilis- is it time to reassess the current prevention guidelines
}

\begin{abstract}
Symptomatic congenital syphilis (SCS) is rare and a nationally notifiable disease reported as a sentinel event. The Center for Disease Control and Prevention has strict guidelines for universal screening of pregnant women for syphilis during the first and third trimesters and at delivery. Whereas, the rate of congenital syphilis had decreased steadily in the United States between the years 2008 and 2012, an increase of $38 \%$ was noted during 2012-2014. This reflects a break in the public health policies for the prevention of maternal-fetal transmission with missed opportunities at different levels. Here, we report a case of symptomatic congenital syphilis presenting with bullous lesions on palm and sole born to a mother who received the recommended antenatal care with strict adherence to the CDC guidelines for prevention. This case is an example of the resurgence of SCS and suggests that the current guidelines for its prevention may need to be redefined with additional and more stringent specifications.
\end{abstract}

Volume 6 Issue $6-2017$

\author{
Snigdha Likki,' Zaineh AljaNini,' Brinda \\ Doraiswamy, ${ }^{2}$ Krishan Kumar, ${ }^{2}$ Rita PVerma ${ }^{2}$ \\ 'Department of Pediatrics, Nassau University Medical Center, \\ USA \\ ${ }^{2}$ Nassau University Medical Center, USA
}

Correspondence: Snigdha Likki, Department of Pediatrics, Nassau University Medical Center, USA, Tel 5129528585, Fax 5165725483,Email slikki@numc.edu

Received: May 26, 2017 | Published: June 5, 2017

\section{Case report}

An appropriate for gestational age Hispanic male infant was born at 37 weeks and 5 days of gestation to a 21 year old primi gravida, who had an uneventful pregnancy until 36 wks of gestation, when her repeat rapid plasma reagent (RPR) test was found to be reactive in the titers of 1:64, along with a positive fluorescent treponemal antibody absorption test (FTA-Abs). Her earlier RPR test performed during the 1 st trimester was non-reactive. The tests for other sexually transmitted diseases and for rectovaginal colonization with group B Streptococcus yielded negative results. The mother denied use of illicit drugs during pregnancy or before. She was treated with 2.4 million units of Benzathine penicillin at the time of diagnosis.

After 12days of maternal treatment the infant delivered vaginally with Apgar scores of 9 at both 1 and 5minutes of life. On physical examination at birth multiple bullous lesions with surrounding erythema were noted on both palms and soles. Some of the bullae ruptured with peeling within few hours of delivery. The infant remained otherwise asymptomatic until day of life 3 when small to moderate amount of mucopurulent non-hemorrhagic nasal discharge appeared. Infant's RPR test was reactive with titers of 1:64 and his FTA-Abs test was positive. The cerebrospinal fluid (CSF) was contaminated with blood with white cell count of $18 / \mathrm{mm}^{3}$, protein of $214 \mathrm{mg} / \mathrm{dl}$ and glucose of $61 \mathrm{mg} / \mathrm{dl}$. The DNA polymerase chain reaction for Treponema Pallidum in CSF was negative. The total serum IgM level was elevated at $250 \mathrm{mg} / \mathrm{dl}$. Rest of the work up, including $\mathrm{C}$ - reactive protein, total and differential white blood cell count, serum electrolytes and kidney and liver function tests was normal. Blood culture sent on admission was negative. Additional work up included long bone x-rays, retinal examination, echocardiography and MRI examination of brain, which were all within normal limits. The infant passed a hearing assessment test. Maternal placental pathology revealed mild to moderate acute chorioamnionitis and mild acute funicitis with a negative Warthin-Starry stain for spirochetes.

The infant was treated with intravenous aqueous crystalline Penicillin $\mathrm{G}$, in the doses of $50000 \mathrm{u} / \mathrm{kg}$ every $12 \mathrm{hrs}$, which was switched to every $8 \mathrm{hrs}$ after 7 days. The treatment continued for a total of 10days. The palm and soles lesions, as well as, the nasal discharge resolved after 3-4days of treatment. He was discharged to home with mother on day of life 11. Father admitted to having penile lesions but tested negative for syphilis. He was, as per guidelines, treated with an intramuscular dose of Benzathine penicillin. As part of the management, ongoing STD prevention education was continued with the parents.

\section{Discussion}

Congenital syphilis (CS) is a nationally notifiable disease and case data from all over the United states including the District of Columbia are reported to CDC. ${ }^{1}$ Occurrence of CS is considered to be a sentinel event reflecting missed opportunities to prevent maternal infection and fetal transmission. The two major opportunities to prevent CS are primary prevention of syphilis among men and women of reproductive age and treatment of pregnant women who are already infected with syphilis. $^{2}$ The US Preventive Services Task Force and the Center for Disease Control and Prevention recommend universal syphilis screening of all pregnant women in the first and third trimester, and at delivery for women at high risk for syphilis. ${ }^{2}$

The rate of congenital syphilis decreased steadily in the United States between the years 2008 and 2012 reaching a nadir of 8.4 cases per 100,000 live births in 2012. ${ }^{3}$ After that, an increase of $38 \%$ was noted during 2012-2014, which coincided with a $22 \%$ national increase in the rates of primary and secondary syphilis among women of reproductive age. ${ }^{3}$ These reports reflected a break in the public health policies for the prevention of maternal syphilis and fetal transmission with missed opportunities at different levels. Among the 458 cases of CS reported in 2014, majority were due to inadequate or no treatment of maternal syphilis. Rest were related to a lack of maternal prenatal care. ${ }^{3}$

Congenital Syphilis results from the transplacental transmission of Treponema pallidum from an infected mother to the fetus. Fetal infection may result in miscarriage, stillbirth, severe illness and even early infant death. ${ }^{3}$ Among those who deliver after 20 weeks of gestation, maternal treatment with penicillin is $98 \%$ effective at prevention if given appropriately and at least 4 weeks or more before birth. ${ }^{4}$ In our case, despite maternal detection at the recommended 
second serological testing and appropriate treatment, fetal transmission and infection could not be prevented as the therapy did not commence fourweeks prior to delivery.

Congenital syphilis is generally asymptomatic. ${ }^{2}$ Among the 458 cases of CS reported in 2014 only $6.5 \%$ of neonates presented with symptoms. Signs and symptoms vary according to the duration of inutero infection and provision of adequate maternal treatment. Among the symptomatic neonates early clinical signs may be cutaneous rash, especially on the palms and soles, rhinitis, nasal snuffles, uveitis, chorioretinitis, generalized lymphadenopathy, hepatosplenomegaly, jaundice, hemolytic anemia, thrombocytopenia, osteochondritis and /or periostitis, failure to thrive and non-immune hydrops fetalis. Our infant exhibited vesiculobullous desquamating rash on palms and soles and hemorrhagic rhinitis as the only presenting features.

There were only 30 cases reported in north east USA in 2014. This is the only symptomatic case recorded in Nassau County in 2016 according to the Nassau County health department. We present this case to draw attention to and reiterate the importance of providing STD prevention education and appropriate testing in pregnant women to prevent maternal infection and fetal transmission of not only syphilis but all other STDs. Acquisition of syphilis late in pregnancy despite adherence to the recommended preventive measures, including appropriate testing and education as in this case, reflects a failure of the system, which might need to be revisited and redefined with additional and more stringent guidelines, such as, on-site testing, same-day treatment, screening of all pregnant women attending emergency clinics and most important, improving access to antenatal care $^{5}$ (Figure 1-4).

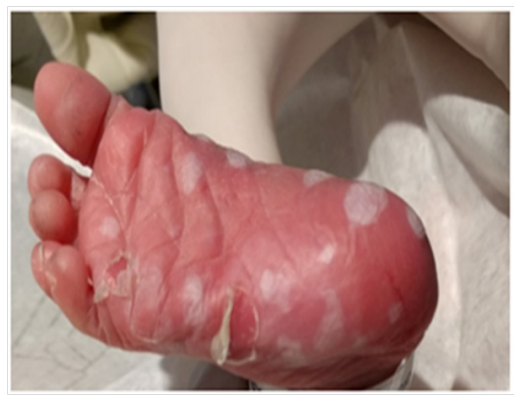

Figure I

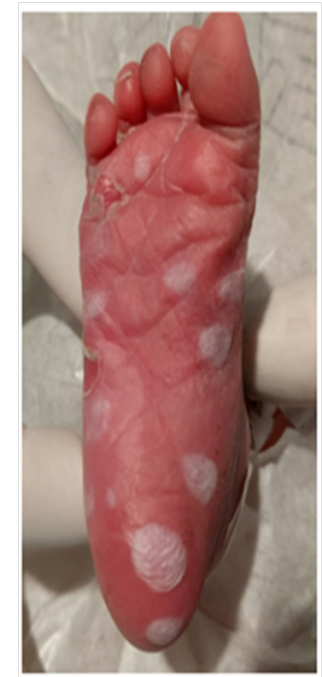

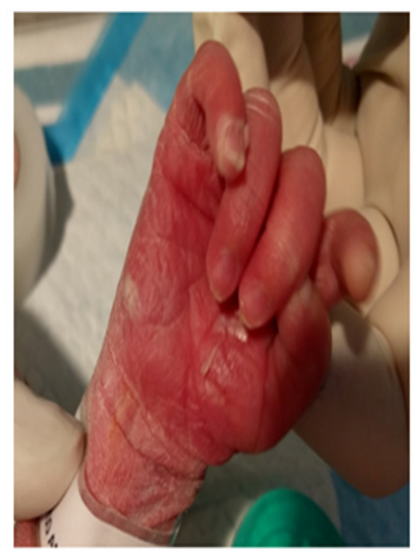

Figure 3

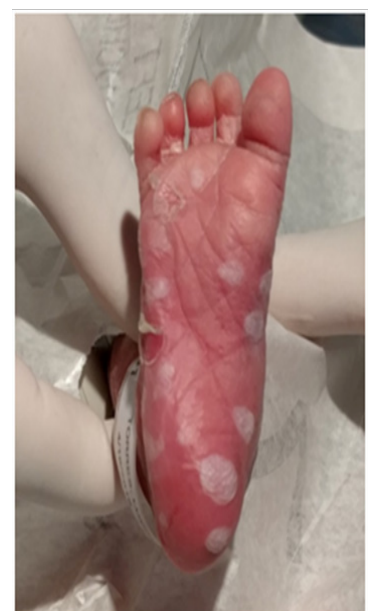

Figure I-4 Echocardiogram showed the presence of $2.5 \times 1.5 \mathrm{~cm}^{2}$ vegetation of the aortic valve.

\section{Acknowledgements}

None.

\section{Conflict of interest}

The author declares no conflict of interest.

\section{References}

1. Bowen $\mathrm{V}, \mathrm{Su}$ J, Torrone E, et al. Morbidity and mortality weekly report. CDC. 2015;64(44):1241-1245.

2. Butterfield R. Pediatrics in review. Syphilis. 2014;35(5):212-213.

3. WHO guidelines for the treatment of Treponema pallidum (syphilis). ISBN. 2016;13:978-924-154980-6.

4. American Academy of Pediatrics. Syphilis. In: Red Book: 2015 Report of the Committee on Infectious Diseases. 30th ed. Kimberlin DW editor. USA: American Academy of Pediatrics; 2015. p. 755-757

5. Warner L, Rochat RW, Fichtner RR, et al. Missed opportunities for congenital syphilis prevention in an urban southeastern hospital. Sex Transm Dis. 2001;28(2):92-98.

Figure 2 